In memoriam

\title{
Nicole-Claude Mathieu: Elle n'est plus
}

\author{
Um discurso "bem formulado" pode ser tão somente o vão refinamento de um discurso vazio, mas \\ assentado sobre a plenitude do poder; uma questão "mal formulada" desvelar um questionamento. . \\ vertiginoso face ao vazio epistemológico. \\ (MATHIEU, L'Anatomie Politique, 1991, p. 140)
}

Faleceu, em 9 de março de 2014, a pesquisadora Nicole-Claude Mathieu, importante militante e teórica do movimento feminista francês. Mathieu era professora da Ecole des Hautes Etudes en Sciences Sociales de Paris (EHESS-Paris) e integrante do Laboratoire d'Anthropologie Sociale (LAS). Seu pensamento, vívido e sagaz, não passava despercebido e influenciou significativamente o debate feminista a partir dos anos 1970, tendo um grande potencial de mobilização, de inspiração, para sxs leitorxs.

É autora de publicações importantes (1991) sobre a construção das categorias "sexo" e "gênero" no âmbito das ciências sociais, que tiveram grande projeção ao denunciar o caráter androcêntrico do uso desse termo nos principais trabalhos etnográficos do século XX. Sua crítica, entretanto, ultrapassa o plano epistemológico. Na década de 1980, momento em que o feminismo lutava por reconhecimento no meio acadêmico na França, Mathieu escrevia que a etnologia de então tratava os homens estudados como seres eminentemente sociais, enquanto relegava às mulheres o lugar da natureza. De modo pertinente, a antropóloga sublinhava o lugar marginalizado concedido às mulheres nas etnografias de então, que raramente apareciam como informantes dxs etnólogxs em trabalhos de campo. Estranhando a ausência da perspectiva das mulheres sobre suas próprias vidas, Mathieu se perguntava se existiria alguma sociedade, ao menos conhecida, na qual as mulheres não falavam. Assim, ela formulava sua crítica epistemológica fundamental: a prática antropológica da época refletia uma "estrutura de pensamento própria à sociedade que a produzia".'

Seu texto clássico sobre a noção de "dominação", publicado em 1985 sob o título "Quand céder n'est pas consentir" ("Quando ceder não é consentir") e republicado em sua coletânea mais importante (1991), marcou gerações de pesquisadoras e pesquisadores. Se a noção de dominação poderia parecer ultrapassada para a crítica antropológica contemporânea, a reflexão de Mathieu ia direto ao ponto em questões candentes de sua época. Em meio ao debate que imbricava pós-colonialidade, Direitos Humanos e relativismo cultural, Mathieu se posiciona politicamente em defesa dos direitos de mulheres de todas as culturas à autonomia sobre seus corpos e consciências. Ao transitar entre as complexidades da categoria "dominação", a autora nos lembra que a noção do consentimento nas relações sexuais comporta uma ambiguidade perversa: "não ceder é uma norma e, ao mesmo tempo, ceder é uma norma". ${ }^{2}$ Não à toa, no caso da violência sexual, a vergonha repousaria invariavelmente sobre a vítima.

1 Nicole-Claude MATHIEU, 1991, p. 17

${ }^{2}$ MATHIEU, 1991, p. 144. 
Seus textos representam uma contribuição essencial ao feminismo e à antropologia, sobretudo no que tange ao debate sobre a emancipação política de sujeitxs marginalizadxs. A sua noção de dominação está refletida na obra de outrxs autorxs fundamentais, e sua crítica à composição androcêntrica da comunidade antropológica de sua época são expressões contumazes da vivacidade de seu pensamento. Mathieu foi uma antropóloga engajada que contribuiu significativamente para a consolidação dos estudos sobre muIheres e feminismo não só na academia francesa, mas também em outros países.

\section{Referências}

FALQUET, Jules. "Pour une anatomie des classes de sexe: Nicole-Claude Mathieu ou la conscience des opprimés." Cahiers du Genre, n. 50, p. 1-25, 2011. Disponível em: < http:// julesfalquet.files.wordpress.com/2010/06/mon-art-ncm-fr.pdf>. Acesso em: 13 mar. 2014.

MATHIEU, Nicole-Claude. L'anatomie politique: catégorisations et idéologies du sexe. Paris: Côté-femmes, 1991.

Vinicius Kauê Ferreira Universidade Federal de Santa Catarina

\section{Publicações de Nicole-Claude Mathieu}

1971 “Notes pour une définition sociologique des catégories de sexe", Epistémologie sociologique (Paris), 1971, 11: 19-39.

REPUBLICATION dans L'Anatomie politique... (cf. Mathieu 1991d): 17-41.

TRADUCTIONS (anglais, serbo-croate)

1974. "Notes towards a sociological definition of sex categories", The Human Context (London), 1974, VI (2): 345-361.

1975-76. "Notes toward a sociological definition of sex categories", International Journal of Sociology (New York), Winter 1975-76, V (4): 14-38. [Revised translation.]

1977. pp. 16-36 in: Mathieu 1977d.

1984. "Zapazanja o socioloskoj definiciji kategorige pola", Vidici (Belgrade), 1984, $n^{\circ}$ 1-2: 52-69.

1973 "Homme-culture et femme-nature? ", L'homme, revue française d'anthropologie (Paris), 1973, XIII (3): 101-113.

REPUBLICATION dans L'Anatomie politique... (cf. Mathieu 1991d): 43-61. TRADUCTIONS (anglais, japonais)

1978. "Man-culture and woman-nature?", Women's Studies International Quarterly (Oxford, U.K.), 1978, I (1): 55-65.

1987. "Ołoko ga bunka de, onna wa shizen ka?" in: Seisa no bunkajinruigaku [Anthropologie et différence des sexes], Tokyo, Shobunsha Publisher, 1987: 59-81.

1974

"Les catégories de sexe en sociologie", pp. 167-178 in: J. Gabel, B. Rousset \& Trinh Van Thao (eds.), L'aliénation aujourd'hui. Paris, Ed. Anthropos, 1974, 217 p. (Publications du Centre universitaire de recherche sociologique d'Amiens, 3). 
1977 a "Paternité biologique, maternité sociale...", pp. 39-48 in: Andrée Michel (ed.), Femmes, sexisme et sociétés. Paris, Presses Universitaires de France, 1977, 208 p. (Sociologie d'aujourd'hui). REPUBLICATION dans L'Anatomie politique... (cf. Mathieu 1991d): 63-73. TRADUCTIONS (espagnol, anglais, italien)

1977 "Paternidad biológica, maternidad social", in A. Michel (ed.), Mujeres, sexismo y sociedad. Madrid, Gráficas Espejo.

1979 "Biological paternity, social maternity : On abortion and infanticide as unrecognized indicators of the cultural character of maternity", pp. 232-240 in: Chris Harris et al. (eds.), The sociology of the family: New directions for Britain. University of Keele (U.K.), 1979, 240 p. (Sociological Review Monographs, 28).

1979. "Paternità biologica, maternità sociale...", pp. 38-47 in: Andrée Michel (a cura di), Donne, sessismo e società. Roma, Armando Armando Editore, 1979, 189 p. (Problemi di sociologia, n.s.).

1984. "Biological paternity, social maternity...", Feminist Issues (Berkeley, CA, USA), Spring 1984, IV (1): 63-71.

$1977 b$ "Masculinité/féminité", Questions féministes (Paris), 1977, 1: 51-67. TRADUCTIONS (anglais)

1977. pp. 1-15 in: Mathieu 1977d.

1980. "Masculinity/femininity", Feminist Issues (Berkeley, CA), 1980, I (1): 51-69.

1977c 3ème partie (pp. 13-18) de "Variations sur des thèmes communs", éditorial de Questions féministes (Paris), 1977, 1. TRADUCTION (anglais)

1980. in Feminist Issues (Berkeley, CA, USA), 1980, I (1).

$1977 \mathrm{~d}$ BROCHURE (en anglais): Ignored by some, denied by others : The social sex category in sociology. London, Women's Research and Resources Centre Publications, 1977, ii +68 . (Explorations in feminism, 2).

1978 a "Où va la répartition des tâches domestiques et éducatives dans la famille?" (interview), Heures claires, mai 1978, 159: 29-34.

1978 b Comptes rendus: de Leïla Sebbar, On tue les petites filles; et de Josée Laure, La cérémonie paternelle, Questions féministes, novembre 1978, 4 : 103-108. TRADUCTION (anglais)

1982

1982. "They kill little girls", Feminist Issues (Berkeley, CA), Spring 1982, II (1) :107-113. "Les mutilations du sexe des femmes", tribune libre (en collab. avec N. Echard), Bulletin de l'Association française des Anthropologues, septembre 1982, 9: 45-49.

$1984 a$ "L'anthropologie des sexes en France" (en collab. avec N. Echard), pp. 176179 in: Actes du Colloque national "Femmes, féminisme et recherches", Toulouse, déc. 1982, Toulouse, AFFER, 1984.

1984b "De la conscience dominée des femmes", Les Cahiers du GRIF, hiver 1984-85, 29 (L'Africaine. Sexes et signes): 7375. 
1985 OUVRAGE COLLECTIF (N.-C. Mathieu, ed.) L'Arraisonnement des femmes. Essais en anthropologie des sexes. Paris, Editions de l'Ecole des Hautes Etudes en Sciences Sociales (EHESS), 1985, 252 p. (collection "Cahiers de l'Homme", n.s., XXIV).

$1985 a$ "Présentation : Femmes, matière à penser... et à reproduire", pp. 5-16 in : N.-C Mathieu (ed.) 1985.

1985b "Quand céder n'est pas consentir. Des déterminants matériels et psychiques de la conscience dominée des femmes, et de quelques-unes de leurs interprétations en ethnologie", pp. 169-245 in: N.-C. Mathieu (ed.) 1985. REPUBLICATION dans L'Anatomie politique... (cf. Mathieu 1991d): 131-225. TRADUCTIONS (anglais, allemand)

1989 et 90 . "When yielding is not consenting. Material and psychic determinants of women's dominated consciousness and some of their interpretations in ethnology “ (Part I), Feminist Issues (Berkeley, CA), Fall 1989, IX (2): 3-49 ; (Part II), Feminist Issues, Spring 1990, X (1): 51-90.

1995. "Nachgeben ist nicht zustimmen. Materielle und psychische Ursachen des dominierten Bewußtseins von Frauen, mit Interpretationen aus der Ethnologie", pp. 41-148 in Mathieu 1995b.

1985c "Critiques épistémologiques de la problématique des sexes dans le discours ethno-anthropologique."

Rapport pour l'UNESCO, Réunion internationale d'experts: "Réflexion sur la problématique féminine dans la recherche et l'enseignement supérieur", Lisbonne, 17-20 sept. 1985. Paris, UNESCO, SHS-85/CONF. 612/6, 61 p. dactyl. PUBLICATION dans L'Anatomie politique... (cf. Mathieu 1991d): 75-127. TRADUCTION (italien)

1989. "Critiche epistemologiche sulla problematica dei sessi nel discorso etnoantropologico “, DWF - Donna/woman/femme (Roma), n 101 1: 8-54.

1986

"Sexe, genre et Nature en anthropologie", Séminaire Limites-Frontières, 21 mars 1986, n 47,19 p. dactyl.

PUBLICATION in Gardey \& Löwy (eds.), L'invention du naturel... ( cf. Mathieu 2000a: 109-124.)

1987 "Femmes du Soi, femmes de l'Autre", pp. 604-614 in : Vers des sociétés pluriculturelles : études comparatives et situation en France. Actes du Colloque international de l'Association française des Anthropologues (AFA), Paris, 9-11 janvier 1986. Paris, Ed. de l'ORSTOM, 1987, 772 p. (Colloques et séminaires). TRADUCTIONS (anglais)

1988. "'Woman" in ethnology : the other of the Other and the other of the Self ", Feminist Issues (Berkeley, CA), Spring 1988, VIII(1): 3-14.

1994. "'Woman' in ethnology ....", The Journal of Social Studies (Dhaka, Bangladesh), October 1994, 66 (special issue on French anthropology): 44-58.

"Identité sexuelle/sexuée/de sexe? Trois modes de conceptualisation du rapport entre sexe et genre", pp. 109-147 in : A.-M. Daune-Richard, M.-C. Hurtig \& M.- 
F. Pichevin (eds.), Catégorisation de sexe et constructions scientifiques. AixenProvence, Université de Provence, 166 p. (Petite collection CEFUP).

REPUBLICATION dans L'Anatomie politique... (cf. Mathieu 1991d): 227- 266. TRADUCTIONS (allemand, anglais, espagnol)

1995. "Das Verhältnis zwischen biologischem und sozialem Geschlecht: Drei Ansätze zur Begriffsbildung", pp. 149-198 in Mathieu 1995b.

1996. "Sexual, sexed and sex-class identities: Three ways of conceptualising the relationship between sex and gender", pp. 42-71 in : Diana Leonard and Lisa Adkins (eds.), Sex in question : French materialist feminism. London, Taylor \& Francis, 1996, viii +216 p. (Gender and Society: Feminist perspectives on the past and present).

2005. "Identidad sexual/sexuada/de sexo? Tres modos de conceptualización de la relación entre sexo y género", pp. 130-175 in: Curiel, Ochy \& Falquet, Jules (compiladoras), El Patriarcadao al desnudo. Tres feministas materialistas. Buenos Aires, Brecha lesbica.

2006. "Tavtotita tou physikou phylou/emphyli/taxis phylou. Tris ennioloyisis tis schesis violoyikou ke kinonikou phylou"', pp.317-373 in: Kostas Yannacopoulos (ed.), Sexoualikotita. Theories ke politikes tis anthropoloyias [Sexualité. Théories et politiques de l'anthropologie]. Athènes, Alexandria.

1990 "Identités de sexe/genre", pp. 42-46 in: Recherches sur les femmes et recherches féministes. Présentation des travaux 1986-1989. Paris, CNRS, $166 \mathrm{p}$.

1991 a (Entrée) "Etudes féministes et anthropologie", pp. 275-278 in: P. Bonte \& M. Izard (eds.), Dictionnaire de l'ethnologie et de l'anthropologie. Paris, Presses Universitaires de France.

1991 b (Entrée) "Différenciation des sexes", pp. 660-664 in: P. Bonte \& M. Izard (eds.), Dictionnaire de l'ethnologie et de l'anthropologie. Paris, Presses Universitaires de France.

$1991 \mathrm{c}$ "Tendances actuelles de la recherche en anthropologie des sexes", pp. 1333 in: Actes du IVème colloque Méga-Tchad (CNRS/ORSTOM, Paris, 14-16 sept. 1988), vol. II: Les relations hommes-femmes dans le bassin du lac Tchad. Textes réunis et présentés par Nicole Echard. Paris, Editions de l'ORSTOM, 329 p. (Colloques et séminaires).

$1991 \mathrm{~d}$ OUVRAGE. L'Anatomie politique. Catégorisations et idéologies du sexe. Paris, Côté-femmes Editions, 296 p. (Recherches).

$1991 \mathrm{e}$ "Sexus/Genus-Identitäten und Frauen-Widerstandsformen (Geschlechtsidentitäten)", Frauen in der Literaturwissenschaft (Universität Hamburg), Juni 1991, 30 ("Frankreich"): 3-8.

REPUBLICATION dans Aspekte feministischer Wissenschaft/ Aspects féministes de la recherche scientifique (9-10 mai 1992). Université de Fribourg, Suisse: 45-64. 
$1991 \mathrm{f} \quad$ "Les transgressions du sexe et du genre à la lumière de données ethnographiques", pp. 69-80 in: M.-C. Hurtig, M. Kail \& H. Rouch (eds.), Sexe et Genre. De la hiérarchie entre les sexes. Paris, Presses du CNRS, 284 p.

1993 "Questions à l'éco-féminisme”, pp. 128-137 in : Maria Inácia d’Avila \& Naumi de Vasconcelos (eds.), Ecologia, feminismo, desenvolvimento. Rio de Janeiro, EICOS/Universidade Federal do Rio de Janeiro, 220 p. (Série Documenta EICOS, 1).

1994a Compte rendu de: S. Shami, L. Taminian et al.: Women in Arab Society. Work Patterns and Gender Relations in Egypt, Jordan and Sudan, L'Homme 130, avril-juin 1994, XXXIV (2): 179-182.

1994 b "'Origines', ou mécanismes de l'oppression des femmes?", in: Catherine Fussinger \& Monique Pavillon (eds.), Femmes, le mauvais genre? (Colloque organisé par le groupe "Regards critiques " à l'Université de Lausanne, 5-6 déc. 1990). Lausanne, Faculté des Lettres, section d'histoire, 1994 (Série Histoire et société contemporaines, $\left.n^{\circ} 16\right)$ : 13-30.

1994c "Dérive du genre/stabilité des sexes", in: Michel Dion (ed.), Madonna. Erotisme et pouvoir. Paris, Editions Kimé, 132 p. (Le sens de l'histoire): 54-70.

1994 d "Problématique de la définition sociologique des catégories de sexe: un itinéraire de recherche", in: Eliane Vogel-Polsky (ed.), Women's studies. Manuel de ressources. (Programme de recherche en sciences sociales, Point d'appui U.L.B.). Bruxelles, Services fédéraux des Affaires scientifiques, techniques et culturelles, sept. 1994: 135-139.

1995 "Relativisme culturel, excision et violences contre les femmes", Sexe et race. Discours et formes nouvelles d'exclusion du XIXe au XXe siècle, 1994-tome 9 (CERIC / Université Paris 7): 87-102.

TRADUCTION (espagnol)

1997. "Relativismo cultural, ablación del clítoris y violencia contra las mujeres ", ARENAL , Revista de historia de las mujeres (Universidad de Granada), vol. 4, $n^{\circ} 1$ (“Mujeres: Cuerpo e identidades"), janvier-juin 1997: 77-94.

1995b OUVRAGE (en allemand) Nachgeben ist nicht Zustimmen. Ethnologische Überlegungen zum Geschlechterverhältnis. Traduit par Christine Hofinger. Introduction par Sabine Strasser. Vienne, Wiener Frauenverlag, 212 p. (Reihe Frauenforschung, Band 30)

1997 a "Le sexe social” (article) + 6 encadrés: "Le 'troisième sexe' chez les Inuit”; "Le 'troisième genre' des berdaches"; "Ni homme, ni femme: les hijras de l'Inde"; "Mariages entre hommes"; "Mariages entre femmes"; "Transsexuels, travestis et transgenre", Sciences et Avenir - Hors Série, n 110 (" Le sexe "), avril/mai 1997: 5257.

REPUBLICATION dans Le sexe, Paris, Maisonneuve et Larose, 1999, 128 p. (coll. QuinteScience): 64-73. 
$1997 \mathrm{~b}$ "Sex and gender in Women's Studies and Diverse Cultures", in: Claudine Raynaud (ed.), Sexualités américaines: regards théoriques, réponses institutionnelles (Communications au Congrès de l'Association Française des Etudes Américaines, 26-28 mai 1995). GRAAT (Publications des groupes de recherches anglo-américaines de l'Université de Tours), $n^{\circ} 17: 27-32$.

1998 a "Banalité du mal et "consentement": des non-droits humains des femmes ", in: Marie-Claire Caloz-Tschopp (ed.), Hannah Arendt, la "banalité du mal" comme mal politique (Actes du Colloque international " Hannah Arendt et le monde d'aujourd'hui : le "droit d'avoir des droits"”, 20-22 mai 1997, Groupe de Genève "Violence et droit d'asile en Europe" et Université Ouvrière de Genève). Paris, L'Harmattan, vol. 2: 162-172.

$1998 \mathrm{~b}$ "Remarques sur la personne, le sexe et le genre", Gradhiva, revue d'histoire ef d'archives de l'anthropologie, $n^{\circ}$ 23: 47-60 (in Dossier "Anthropologie des sexes", réuni et présenté par N.-C. Mathieu, pp. 47-126).

1999 "Bourdieu ou le pouvoir auto-hypnotique de la domination masculine", Les Temps Modernes, n 604, mai-juin-juillet 1999 : 286-324.

2000a "Les sexes et la "nature" chez les ethnologues et les ethnologisés (rappel historique)", in: Delphine Gardey \& Ilana Löwy (eds.), l'Invention du naturel. Les sciences et la fabrication du féminin et du masculin. Paris, Editions des archives contemporaines, 227p. (Histoire des sciences, des techniques et de la médecine): 109-124.

2000b "Anthropologie et 'homosexualités', in: Martine Gross (ed.), Homoparentalités: Ełat des lieux. Parentés et différence des sexes. Paris, ESF éditeur, 304 p. (La vie de l'enfant): 89-94.

2000 (Entrée) "Sexe et genre", in: Dictionnaire critique du féminisme, Helena Hirata, Françoise Laborie, Hélène Le Doaré \& Danièle Senotier (eds.). Paris, PUF. (Politique d'aujourd'hui): 191-200.

TRADUCTIONS (espagnol, japonais)

2002. "Sexo y género", in: H. Hirata, F. Laborie, H. Le Doaré, D. Senotier (coordinadoras), Diccionario crítico del feminismo. Madrid, Sintesis: 236-244.

2002. In: H. Hirata, F. Laborie, H. Le Doaré, D. Senotier (eds), Josei Gaku [Science des femmes], Tokyo, Fujiwara Shoten (coll. "Jitem Yomu" [Lire le dictionnaire]), 458 p.

2000d "Un hommage critique à Lévi-Strauss et Freud : Gayle Rubin (1975)". Entretien réalisé par C. Quiminal, Journal des Anthropologues, n 82-83 ("Anthropologie des sexualités"): 41-52.

2003 "La femme des hordes primitives selon Simone de Beauvoir", Europaea (Université de Cagliari, Italie), $\mathrm{n}^{\circ}$ 1/2: 45-56.

2004 a "Les hordes primitives", in: Ingrid Galster (ed.), Simone de Beauvoir: Le Deuxième sexe. Le livre fondateur du féminisme moderne en situation. Paris, Éditions Honoré Champion: 87-102. 
2004 b "Matriarcat" ou résistance? Mythes et réalités», Revue Espace lesbien , $\mathrm{n}^{\circ} 4$ (Toulouse, Bagdam Espace Édition): 73-84.

2007a (Entrée) « Féminin - féminin et masculin », in : Michela Marzano (dir.), Dictionnaire du corps, Paris, PUF (Quadrige/Dicos poche): 379-384.

2007b (Sous la dir.de) Une maison sans fille est une maison morte. La personne et le genre en sociétés matrilinéaires et/ou uxorilocales. Paris, Éditions de la Maison des Sciences de l'Homme, vii + $503 \mathrm{p}$.

2007c "Introduction: Circulation des hommes, permanence des femmes, matriarcats imaginaires et autres curiosités...", in ibid.: 1-54.

2007d «Glossaire » (en collab. avec M. Gestin et F.-M. Renard Casevitz), in: ibid.: 493-503.

\section{Ouvrages traduits}

Gayle RUBIN, " The traffic in women: Notes on the "political economy" of sex", in Reiter, Rayna R., Toward an anthropology of women, New York, Monthly Review Press, 1975: L'économie politique du sexe: Transactions sur les femmes et systèmes de sexe/genre, traduit de l'anglais par Nicole-Claude Mathieu avec la collaboration de Gail Pheterson, Les Cahiers du CEDREF (Université Paris 7), $n^{\circ} 7,1998,82 p$.

Gail PHETERSON, The Prostitution Prism (Amsterdam University Press, 1996): Le Prisme de la prostitution. Édition augmentée. Traduit de l'anglais (ÉtatsUnis) par Nicole-Claude Mathieu. Paris, L'Harmattan, 2001 (coll. "Bibliothèque du féminisme"), $214 \mathrm{p}$. 